\title{
Numerical and Experimental Investigation of a Fiber-Optic Sensor Consisting of a Fiber Bragg Grating in a Two-Mode Fiber for Simultaneous Sensing of Temperature and Strain
}

\author{
A. Siekiera ${ }^{1,2}$, R. Engelbrecht ${ }^{1,2}$, L. Buethe ${ }^{1}$, B. Schmauss ${ }^{1,2}$ \\ ${ }^{1}$ Institute of Microwaves and Photonics, University of Erlangen-Nuremberg, Cauerstr. 9, 91058 \\ Erlangen, Germany, \\ Alexander.Siekiera@fau.de \\ ${ }^{2}$ Erlangen Graduate School in Advanced Optical Technologies (SAOT), University of Erlangen- \\ Nuremberg, Paul-Gordan-Str. 6, 91052 Erlangen, Germany
}

\begin{abstract}
We investigate a fiber-optic sensor for the simultaneous measurement of temperature and strain. It consists of a fiber Bragg grating (FBG) inscribed into a short piece of two-mode fiber (TMF) that is spliced in-between a standard single mode fiber (SMF). By monitoring the spectral shift of the Bragg wavelength and the mode interference (MI) pattern, both physical quantities can be determined simultaneously. The sensor response to temperature and strain was investigated theoretically using a numerical sensor model and the results were verified experimentally. The measured temperature and strain sensitivities of the $\mathrm{Ml}$ are found to be $175,3 \mathrm{pm} / \mathrm{K}$ and $3,24 \mathrm{pm} / \mu \varepsilon$, which is large compared to the FBG having sensitivities of $9,6 \mathrm{pm} / \mathrm{K}$ and $1,04 \mathrm{pm} / \mu \varepsilon$. The simultaneous measurement of temperature and strain was tested experimentally yielding promising results with maximum errors of measurement of $1,5 \mathrm{~K}$ and $82 \mu \varepsilon$ respectively.
\end{abstract}

Key words: Fiber optic sensor, fiber Bragg grating, mode interference, simultaneous measurement, sensor model.

\section{Introduction}

Fiber Bragg gratings (FBG) are important fiberoptic sensor transducers, yet their crosssensitivity to temperature $T$ and strain $\varepsilon$ often is a challenge in practical applications. Proper packaging of the FBG can reduce strain influences in a temperature measurement, but for strain measurements a second reference temperature sensor usually is necessary. Several different compensation methods have been proposed and investigated [1]. We recently proposed a FBG inscribed in a twomode fiber for the simultaneous measurement of $T$ and $\varepsilon$, and we refer to it as FBG-MI-sensor [2]. In this paper theoretical results that were obtained from a numerical model are presented and compared to experimental results, showing the potential of the proposed sensor for simultaneous measurement of $T$ and $\varepsilon$.

\section{Principle of the FBG-MI-Sensor}

The examined sensor transducer is shown in Fig. 1. A FBG is inscribed into a short piece of two-mode fiber (TMF, SM2000). For the simulations and experiments presented in the paper the length of the TMF is $20 \mathrm{~cm}$. By splicing the TMF in-between a standard single mode fiber (SMF) with a lateral offset of $\Delta x=5,3 \mu \mathrm{m}$, both modes $\mathrm{LP}_{01}$ and $\mathrm{LP}_{11}$ are excited at the first splice with approximately the same power coupling efficiency. Both modes propagate along the TMF with different propagation constants $\beta_{01}$ and $\beta_{11}$, resulting in a
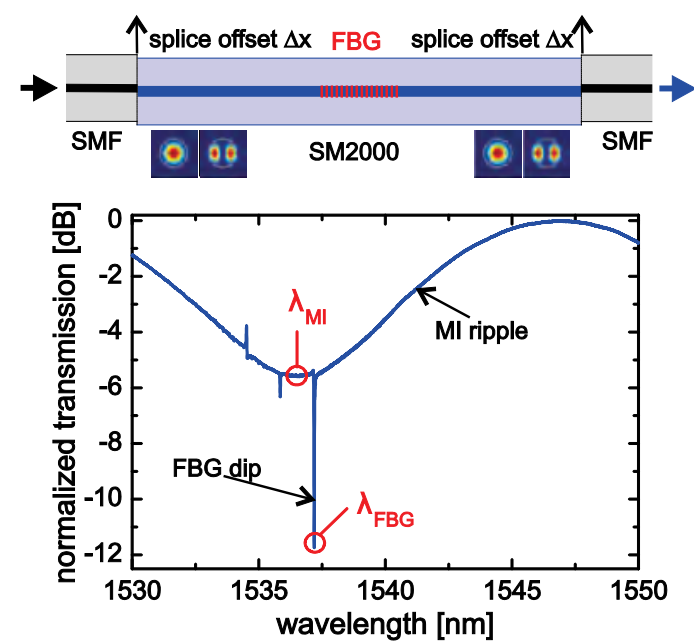

Fig. 1. Principle setup of the FBG-MI-sensor and measured transmission spectrum. 
wavelength dependent phase difference at the second splice. Thus a periodic mode interference $(\mathrm{MI})$ ripple pattern is visible in the transmission spectrum shown in Fig. 1. As the $\mathrm{MI}$ ripple pattern is spectrally shifted if the fiber is subject to temperature changes or strain, the wavelength $\lambda_{M I}$ of a $\mathrm{MI}$ minimum (or maximum) can be used as a first measurand for the simultaneous measurement of temperature and strain. The strong dip in the transmission spectrum of Fig. 1 at $\lambda_{F B G}$ is due to the reflection of the $L P_{01}$ mode at the FBG and is used as the second measurand. By monitoring the spectral shifts $\Delta \lambda_{M I}$ and $\Delta \lambda_{F B G}$ the temperature change $\Delta T$ and fiber strain $\varepsilon$ can be calculated from

$$
\left(\begin{array}{c}
\Delta \lambda_{F B G} \\
\Delta \lambda_{M I}
\end{array}\right)=\left(\begin{array}{cc}
K_{F B G, \varepsilon} & K_{F B G, T} \\
K_{M I, \varepsilon} & K_{M I, T}
\end{array}\right) \cdot\left(\begin{array}{c}
\varepsilon \\
\Delta T
\end{array}\right),
$$

if the respective temperature and strain sensitivities $K_{F B G, T}, K_{F B G, \varepsilon}, K_{M I, T}$ and $K_{M I, \varepsilon}$ of the FBG-MI-sensor are known and the matrix in eq. (1) is invertible.

\section{Sensor Model and Numerical Results}

To gain theoretical insight into the sensor properties, the FBG-MI-sensor was modeled numerically. The mode-fields of the SMF (core diameter $8,2 \mu \mathrm{m}$, Germanium mole fraction $3,5 \%$ ) and the TMF (SM2000, core diameter $11,2 \mu \mathrm{m}$, Germanium mole fraction 3,75\%) are calculated using the weakly guiding fiber approximation of linearly polarized (LP) modes [3]. The mode specific propagation constants and the chromatic dispersion were considered using the Sellmeier equation with coefficients for $\mathrm{SiO}_{2}$ and $\mathrm{GeO}_{2}$ from literature [4]. The complex transmission coefficient $t_{F B G}(\lambda)$ of the FBG is calculated using the transfer-matrixmethod [5]. From these calculations the transmission spectrum of the whole sensor structure can be determined numerically. The influence of temperature and axial strain is included in the sensor model by considering the change of basic physical parameters, namely the TMF core diameter, the refractive indices of core and cladding, the TMF length and the grating period of the FBG.

In Fig. 2 the calculated transmission spectra are shown exemplary for three different values of $\Delta T$ and zero fiber strain, illustrating the spectral shift of $\lambda_{M I}$ and $\lambda_{F B G}$. The MI ripple period $\Lambda_{M I}$ in the simulations was $19,8 \mathrm{~nm}$ for a TMF length of $20 \mathrm{~cm}$. It represents the maximum wavelength shift $\Delta \lambda_{M I}$ for which unambiguous measurement results can be obtained. The theoretical sensor response curves are shown in Fig. 3 together with the temperature and strain sensitivities that were found from linear

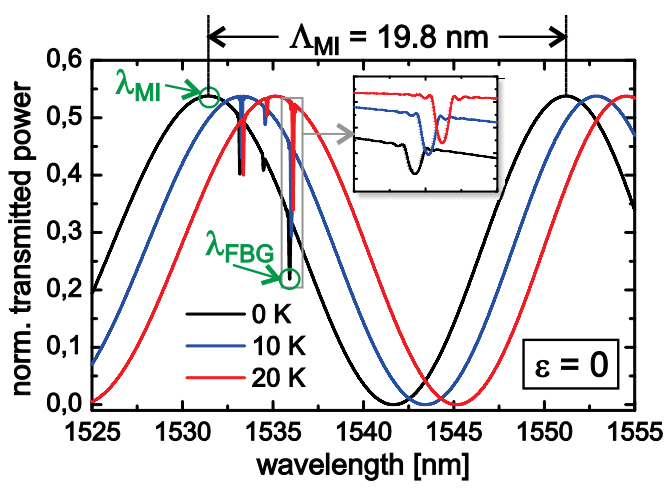

Fig. 2. Calculated transmission spectra of the FBG$\mathrm{MI}$-sensor for different temperature changes.
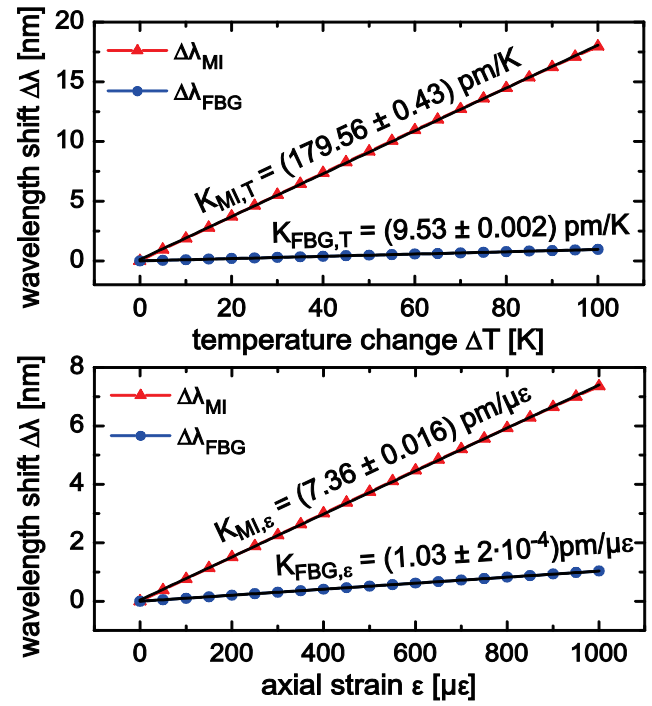

Fig. 3. Simulated sensor response and corresponding temperature and strain sensitivities.

fits to the simulation data. The temperature and strain sensitivities of the FBG are in good agreement with the expected values from literature [6]. For the MI both sensitivities have larger values compared to the FBG, with a remarkably high temperature sensitivity of $179,56 \mathrm{pm} / \mathrm{K}$. From the values given in Fig. 3 the determinant of the sensitivity matrix in eq. 1 is found to be nonzero, thus eq. 1 can be solved for $\varepsilon$ and $\Delta T$.

A simulation of the $\mathrm{Ml}$ ripple spectrum over a large wavelength span is shown in Fig. 4 for three different conditions for $\Delta T$ and $\varepsilon$. Due to the chromatic dispersion of the TMF the ripple period $\Lambda_{M I}$ is not constant over wavelength, but is increasing towards a critical wavelength [7], which for the examined fiber is located at $\lambda_{c}=1400 \mathrm{~nm}$. When comparing the spectral shifts of the MI maxima in the marked areas (I IV) in Fig. 4, it is obvious that also the sensitivities $K_{M I, T}$ and $K_{M I, \varepsilon}$ are varying over wavelength with strongly increasing sensitivities towards the critical wavelength. From numerical simulations it was found that the temperature and strain sensitivity changes from $228 \mathrm{pm} / \mathrm{K}$ to 


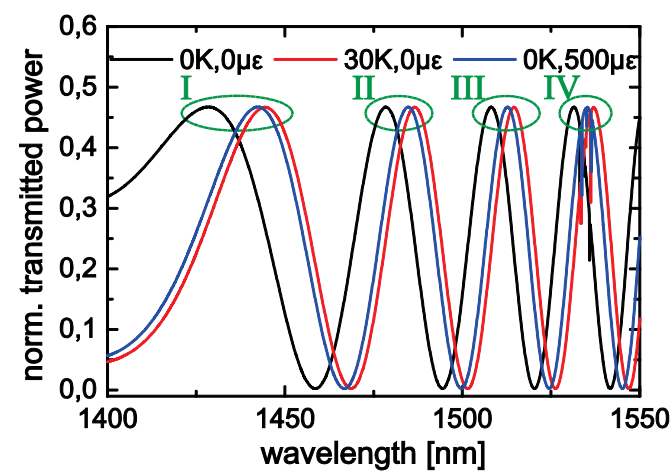

Fig. 4. MI spectrum over large wavelength span.

$137 \mathrm{pm} / \mathrm{K}$ and $10,0 \mathrm{pm} / \mu \varepsilon$ to $4,6 \mathrm{pm} / \mu \varepsilon$ within a wavelength interval of $1500 \mathrm{~nm}$ to $1600 \mathrm{~nm}$. For a given sensor this means that the temperature sensitivity is depending on the actual value of the fiber strain and vice versa. As a consequence for the simultaneous measurement of temperature and strain, a 2-D calibration of the Ml sensitivities over the full measurement range for $\Delta T$ and $\varepsilon$ would be necessary in order to avoid large calibration errors. As an alternative to the evaluation of the MI wavelength shift $\Delta \lambda_{M I}$, the phase shift $\Delta \varphi_{M I}$ of the $\mathrm{Ml}$ ripple spectrum can be evaluated, strongly mitigating the problem of wavelength dependent sensitivities of the Ml that is shown in Fig. 4. The method is based on phase extraction from the measured $\mathrm{MI}$ ripple spectrum using the fast Fourier transform (FFT) [8]. From numerical simulations the temperature and strain sensitivity of the Ml defined by the phase shift $\Delta \varphi_{M I}$ were found to be linear with values of $K_{M I, T}=55,1 \mathrm{mrad} / \mathrm{K}$ and $K_{M I, \varepsilon}=2,2 \mathrm{mrad} / \mu \varepsilon$. The corresponding sensor response curves are shown in Fig. 5.
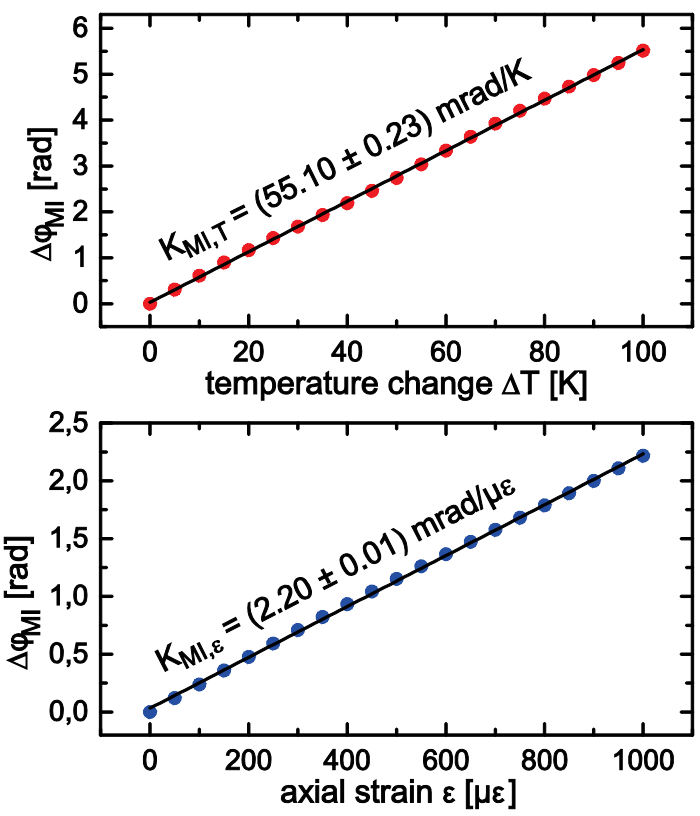

Fig. 5. Simulated MI sensor response for evaluation of the phase change $\Delta \varphi_{M I}$.

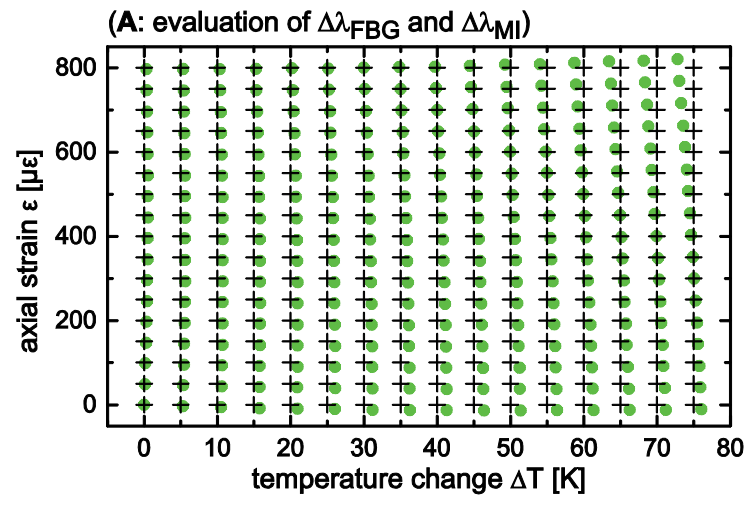

(B: evaluation of $\Delta \lambda_{\mathrm{FBG}}$ and $\Delta \varphi_{\mathrm{MI}}$ )

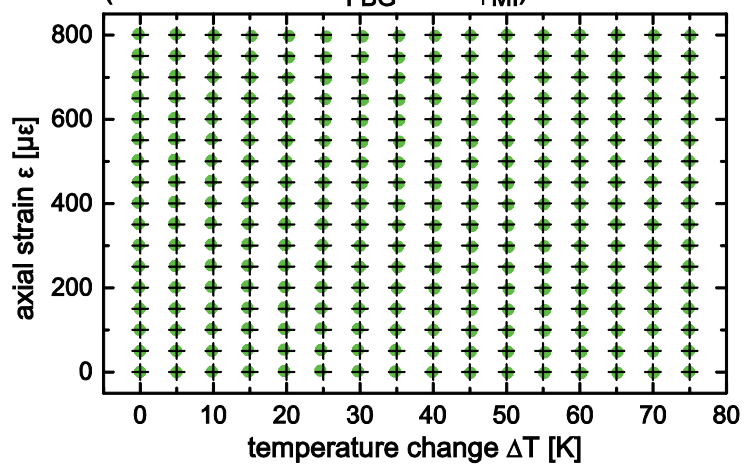

Fig. 6. Simulation of the sensor response for simultaneous measurement of temperature change and strain for different demodulation methods $A$ and $B$ (cross: preset value, circle: recovered value).

The performance of both demodulation methods (A: evaluation of $\Delta \lambda_{F B G}$ and $\Delta \lambda_{M I}$, B: evaluation of $\Delta \lambda_{F B G}$ and $\Delta \varphi_{M I}$ ) was investigated numerically by simulating the simultaneous measurement of temperature change and strain. In both cases the linear calibration of the temperature and strain sensitivities of FBG and $\mathrm{Ml}$ is performed with reference to $\varepsilon_{0}=0 \mu \varepsilon$ and $\Delta T_{0}=0 \mathrm{~K}$. Fig. 6 shows the results for both demodulation methods $A$ and $B$ regarding the agreement between the preset and the recovered values for $\Delta T$ and $\varepsilon$. As was explained before quite large calibration errors may occur for method $A$, due to the wavelength dependence of $K_{M I, T}$ and $K_{M I, \varepsilon}$. The maximum calibration errors for the examined measurement range are $2,2 \mathrm{~K}$ for temperature and $20,6 \mu \varepsilon$ for strain measurements. Using the demodulation method B significantly reduces the calibration errors as is illustrated in the lower graph of Fig. 6. Here the maximum calibration errors are $0,4 \mathrm{~K}$ and $4,0 \mu \varepsilon$ for temperature and strain respectively.

\section{Experimental Results}

A FBG-MI-sensor was fabricated by inscribing a FBG into the TMF with a length of $L=20 \mathrm{~cm}$. The experimental setup for the temperature and strain calibration is shown in Fig. 7. The sensor 


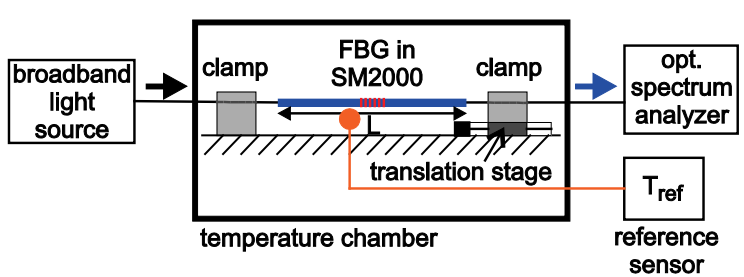

Fig. 7. Experimental setup.

fiber is mounted in two magnetic clamps, one being fixed while the other can be moved by a translation stage to apply defined axial strain to the fiber. The setup is placed in a temperature chamber which can adjust the temperature in a range from $-75^{\circ} \mathrm{C}$ to $180^{\circ} \mathrm{C}$. The amplified spontaneous emission of an Erbium-doped fiber amplifier is used as a broadband light source and the transmission spectrum is recorded by an optical spectrum analyzer (OSA) with a resolution bandwidth of $0,02 \mathrm{~nm}$ and a wavelength discretization step size of $2 \mathrm{pm}$. A reference temperature sensor (repeatability standard deviation $\sigma_{r}=0,05 \mathrm{~K}$ ) is used to measure the actual temperature in the chamber. Fig. 8 shows the measured transmission spectra of the FBG-MI-sensor for different values of $\Delta T$. The results show a very good agreement with the simulated spectra in Fig. 2 regarding the ripple period $\Lambda_{M I}$, which is $21 \mathrm{~nm}$ for the experiment and $19,8 \mathrm{~nm}$ for the simulations, and the spectral shift of the MI wavelength $\lambda_{M I}$ and the Bragg wavelength $\lambda_{F B G}$.

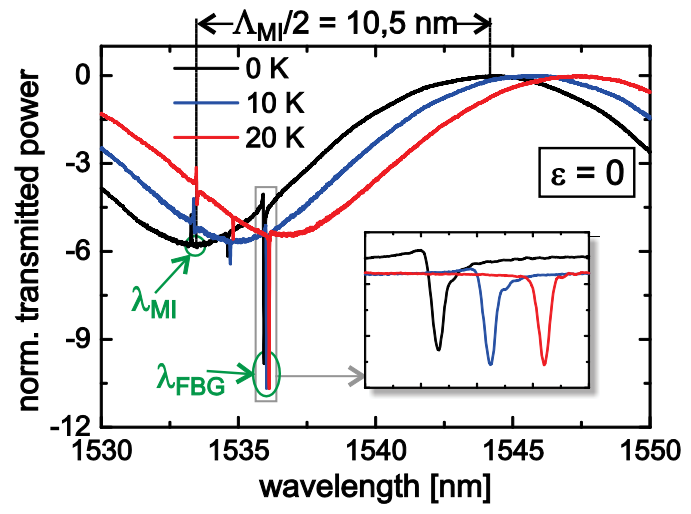

Fig. 8. Measured transmission spectra of the FBGMI-sensor for different temperature changes.

The sensor response of the FBG-MI-sensor was measured for temperatures ranging from $25^{\circ} \mathrm{C}$ to $60^{\circ} \mathrm{C}$ and axial strain of $0 \mu \varepsilon$ to $1000 \mu \varepsilon$. The measurement results are shown in Fig. 9 together with the sensitivities that were found from the linear fits to the data. Except for the smaller value of the $\mathrm{Ml}$ strain sensitivity $K_{M I, \varepsilon}$ the experimental results are in excellent agreement with the simulated sensor response in Fig. 3. The deviation for $K_{M I, \varepsilon}$ is supposed to result from lacking information about the exact fiber parameters, which makes the optimization of all simulation parameters in the sensor model
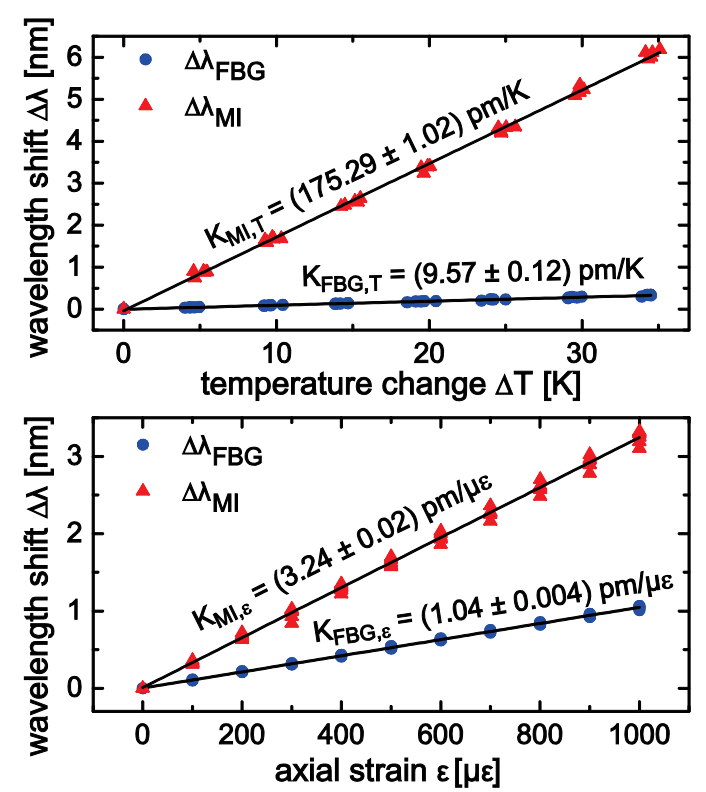

Fig. 9. Measured FBG-MI-sensor response.

difficult. Yet to get insight into the basic sensor properties this deviation is negligible.

The wavelength dependence of the MI sensitivities was investigated exemplary for the temperature sensitivity $K_{M I, T}$. The $\mathrm{Ml}$ ripple spectrum was recorded over a wavelength span of $100 \mathrm{~nm}$ at different temperatures and the wavelength shifts of the multiple MI maxima and minima were evaluated, yielding the temperature sensitivities at the respective wavelengths. In Fig. 10 the measured sensitivities are plotted together with the simulated curve that was determined from the numerical sensor model. Within the measured wavelength span the temperature sensitivity changes from $185,3 \mathrm{pm} / \mathrm{K}$ at $1523 \mathrm{~nm}$ to $135,4 \mathrm{pm} / \mathrm{K}$ at $1582 \mathrm{~nm}$. As a consequence by changing the interrogation wavelength the temperature sensitivity of the Ml-sensor can be adjusted to very high values.

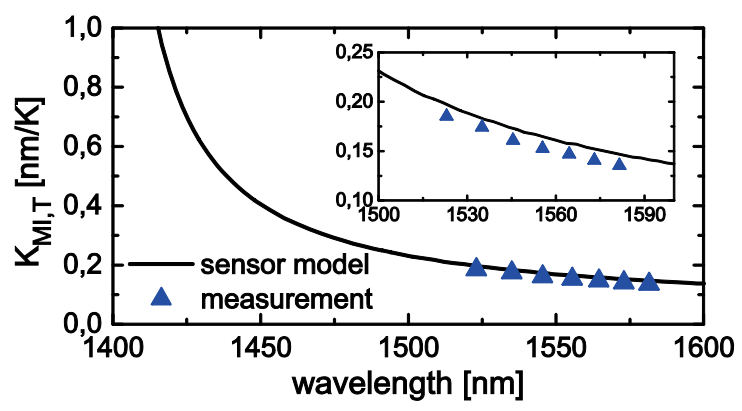

Fig. 10. Theoretical and measured wavelength dependence of the MI temperature sensitivity.

\section{Simultaneous Measurement Experiment}

The potential of the FBG-MI-sensor for simultaneous measurements of temperature and strain was tested by applying both temperature changes and axial fiber strain to 
the sensor fiber. For that purpose the FBG-MIsensor was mounted in the clamping setup of Fig. 7 with a certain amount of pre-strain applied to the fiber. Due to the large thermal expansion coefficient of the aluminum base plate of the clamping setup $\left(\alpha_{A l} \approx 21,1 \cdot 10^{-6} 1 / \mathrm{K}\right)$ compared to the fiber $\left(\alpha_{\mathrm{SiO} 2} \approx 0,55 \cdot 10^{-6} 1 / \mathrm{K}\right)$, the FBG-MI-sensor is subject to additional strain when the temperature chamber is heated. To distinguish between thermally induced strain and applied axial strain in the experiment, the effective temperature sensitivity of the FBG and MI were measured. The results listed in table 1 expectedly show larger values for the effective temperature sensitivities in case of a prestrained sensor fiber.

Tab. 1: Effective temperature sensitivity and strain sensitivity of the pre-strained FBG-MI-sensor.

\begin{tabular}{|c|c|c|}
\hline & FBG & MI \\
\hline Eff. temp. sens. [pm/K] & 33,5 & 232 \\
\hline Strain sens. $[\mathrm{pm} / \mu \varepsilon]$ & 1,04 & 3,24 \\
\hline
\end{tabular}

From the measured wavelength shifts $\Delta \lambda_{F B G}$ and $\Delta \lambda_{M I}$ the applied fiber strain $\varepsilon$ and temperature change $\Delta T$ were calculated by solving eq. 1. The results of the simultaneous measurement experiment are shown in Fig. 11, where the black squares indicate the preset values for temperature changes $\Delta T$ and fiber strain $\varepsilon$ and the red circles indicate the measured values.

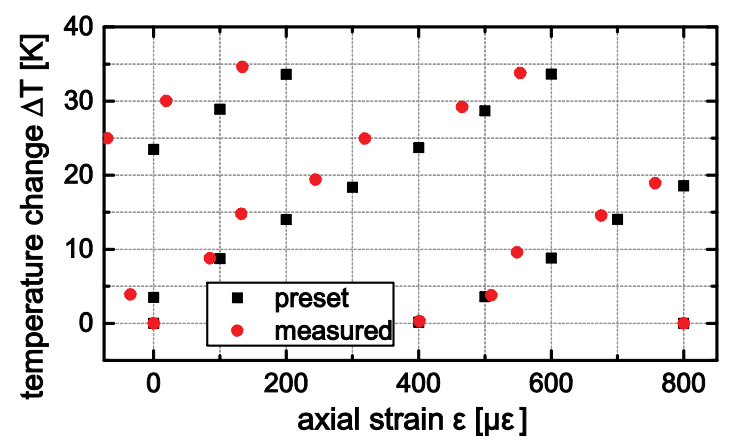

Fig. 11. Results for the simultaneous measurement of temperature and strain.

The measured temperature changes are in very good agreement with the preset values with a maximum deviation of $1,5 \mathrm{~K}$. The measurement results for strain seem to underestimate the preset values, which might be attributed to a systematic error caused by the clamping setup used in the experiment. A slight slipping of the glass fiber relative to the clamped polymer coating could be observed, causing the quite large deviations between preset and measured strain values. The maximum deviation was $82 \mu \varepsilon$. From the functional relation resulting from eq. 1 between the measurands $(\Delta T, \varepsilon)$ and the measured values of $\Delta \lambda_{F B G}$ and $\Delta \lambda_{M I}$ and the standard uncertainties for the wavelength measurement and the temperature and strain sensitivities, the combined uncertainty of measurement $u(\Delta T)$ and $u(\varepsilon)$ can be calculated [9]. Taking the wavelength standard uncertainty of the OSA of $5 \mathrm{pm}$ and the standard uncertainties of the temperature and strain sensitivities given in Fig. 9, the combined measurement uncertainties are estimated to be $u(\Delta T)=0,77 \mathrm{~K}$ and $u(\varepsilon)=17,1 \mu \varepsilon$.

\section{Discussion}

The combination of a FBG- and a Ml-sensor and its potential for the simultaneous measurement of temperature and strain were investigated. A numerical sensor model of the FBG-MI-sensor was developed, permitting the theoretical examination of the sensor properties. The simulation results for the temperature and strain response of the FBGMI-sensor show the potential for simultaneous measurement of both quantities. The theoretical results also point out the importance of the wavelength dependence of the MI ripple period $\Lambda_{M I}$ and the varying temperature and strain sensitivities of the Ml-sensor over wavelength. To avoid the requirement of complex 2-D calibrations over the full measurement range for temperature change and strain, the phase shift $\Delta \varphi_{M I}$ is evaluated instead of the wavelength shift $\Delta \lambda_{M I}$. Numerical investigations approve the significantly reduced calibration errors for the FFT demodulation method compared to the method of evaluating the MI wavelength shift.

The presented experimental results are in very good agreement with numerical results. The temperature and strain sensitivities of the FBGMl-sensor were experimentally found to be $K_{M I, T}=175,3 \mathrm{pm} / \mathrm{K}, \quad K_{F B G, T}=9,6 \mathrm{pm} / \mathrm{K}$, $K_{M I, \varepsilon}=3,24 \mathrm{pm} / \mu \varepsilon$ and $K_{F B G, \varepsilon}=1,04 \mathrm{pm} / \mu \varepsilon$. The measurement uncertainties for temperature and strain are estimated to be $0,77 \mathrm{~K}$ and $17,1 \mu \varepsilon$. The FBG-MI-sensor was tested in a simultaneous measurement experiment, yielding very promising results. Both temperature change and fiber strain could be measured simultaneously by evaluating the wavelength shifts $\Delta \lambda_{F B G}$ and $\Delta \lambda_{M I}$ with maximum absolute deviations of $1,5 \mathrm{~K}$ and $82 \mu \varepsilon$. The measurement errors could be further reduced by optimizing the clamping setup in the experiment. Due to the very high temperature sensitivity the FBG-MI-sensor has great potential as a single-sensor for applications with demanding requirements considering the temperature resolution and the suppression of strain cross-sensitivity. 


\section{Acknowledgments}

This work was funded by the Bavarian Research Foundation (BFS) within the SpeziFBG project (AZ-900-09) and the Erlangen Graduate School in Advanced Optical Technologies (SAOT) by the German National Science Foundation (DFG) in the framework of the German excellence initiative. The authors would like to thank Dr. T. Bosselmann, Dr. M. Willsch and M. Villnow from Siemens CT (Erlangen, Germany) for valuable discussions and collaboration within the SpeziFBG project.

\section{References}

[1] A. Othonos, K. Kalli, Fiber Bragg Gratings Fundamentals and Applications in Telecommunication and Sensing, chap. 7.4, 330-337, Artech House (1999).

[2] A. Siekiera, R. Engelbrecht, L. Buethe, and B. Schmauss, Simultaneous Sensing of Temperature and Strain by Combined FBG and Mode-Interference Sensors," in Bragg Gratings, Photosensitivity, and Poling in Glass Waveguides, OSA Technical Digest (online), Optical Society of America (2012), paper BTu2E.6.

[3] D. Gloge, Weakly Guiding Fibers, Appl. Opt. 10, 2252-2258 (1971).

[4] J. W. Fleming, Dispersion in $\mathrm{GeO}_{2}-\mathrm{SiO}_{2}$ Glasses, Appl. Opt. 23, 4486-4493 (1984).

[5] R. Kashyap, Fiber Bragg Gratings, chap. 4.8, 171-177, Academic Press (2010).

[6] A. D. Kersey, et al., Fiber grating sensors, J. Lightw. Technol. 15, 1442-1463 (1997); doi: $10.1109 / 50.618377$.

[7] S. M. Tripathi, A. Kumar, E. Marin, J.-P. Meunier, Critical Wavelength in the Transmission Spectrum of SMS Fiber Structure Employing $\mathrm{GeO}_{2}$-Doped Multimode Fiber, IEEE Photon. Technol. Lett. 22, 799-801 (2010); doi: 10.1109/LPT.2010.2046155.

[8] D. Barrera, et al., Low-Loss Photonic Crystal Fiber Interferometers for Sensor Networks, J. Lightw. Technol. 28, 3542-3547 (2010); doi: 10.1109/JLT.2010.2090861.

[9] J.-L. Tang, J.-N. Wang, Error Analysis and Measurement Uncertainty for a Fiber Grating Strain-Temperature Sensor, Sensors 10, 65826593 (2010); doi: 10.3390/s100706582. 